УДК 159.923

DOI 10.23951/2307-6127-2020-1-174-181

\title{
ПСИХОСОМАТИЧЕСКИЕ ЗАБОЛЕВАНИЯ КАК ПОИСК СОБСТВЕННОГО Я
}

\section{T. А. Ребеко}

\section{Институт психологии РАН, Москва}

Обсуждается проблема психосоматических заболеваний. Кожные заболевания рассматриваются как общепсихологическая модель дифференциации и интеграции знаний о собственном я. В работе исследуется сопряженная связь между психосоматическими кожными заболеваниями (псориаз и атопический дерматит) и репрезентацией собственного я. Приводятся литературные данные в пользу утверждения о том, что ранние отношения матери и младенца являются критическими для последующего психического развития. Рассматривается понятие «первичная кожа» (Э. Бик), которая выполняет функцию связывания («слипания») частей личности ребенка. Высказывается предположение о том, что опыт, который переживает младенец в ранних симбиотических отношениях с матерью, является прототипом для последующих моделей взаимодействия человека с окружающим миром. Если ребенок сталкивается с ранним травмирующим опытом материнского «непонимания», то он оказывается неспособным к интеграции «того, что внутри, и того, что происходит вовне». Рассматриваются понятия «вторая кожа» и «вторичный симбиоз» как защитные механизмы, возникающие в случае травматического опыта сепарации. Соотносятся формы вторичного симбиоза с двумя типами взгляда матери - невидящим и искажающим. Предполагается, что два типа взглядов и форм вторичного симбиоза соотносятся с атопическим дерматитом и псориазом. Высказывается гипотеза о том, что в группе «атопический дерматит» выражены дефекты, сопряженные с затянувшимся симбиозом (т. е. с невидящим взором матери), а в группе «псориаз» - с несостоявшимся или преждевременно разорванным симбиозом (т. е. с искажающим взглядом матери). Проведено исследование с помощью методики Лыбко (репрезентация себя посредством конструктов «я сам»/《я для другого) и методики «Крем» (представление о поверхности кожи с помощью разных конструктов крема). В исследовании участвовало 72 женщины в возрасте 20-40 лет (медиана - 37 лет), из них 30 женщин - здоровых (НОРМ), 17 - страдающих псориазом (ПС) и 25 - атопическим дерматитом (АД). Методом непараметрического анализа (критерий Краскала - Уоллиса) доказывается, что репрезентация истинного я проявляется как тенденция к слиянию/инкапсуляции в разных группах испытуемых. Кожные заболевания (АД и ПС) отражают разные способы преодоления вторичного симбиоза. При репрезентации собственного я группа АД демонстрирует тенденцию к слиянию, а группа ПС - к избеганию.

Ключевые слова: собственное я, психическая кожа, вторая кожа, первичный/ вторичный симбиоз, атопический дерматит/псориаз, тенденция к слиянию/инкапсуляцุич.

Научный интерес к психосоматическим заболеваниям происходит вследствие того, что современная наука все более явно свидетельствует о связи опыта телесности с процессом становления психического. Проблема психосоматических заболеваний постепенно 
смещается из области клинических исследований в область нейронауки [1, с. 80]. Все большее внимание исследователи уделяют ранним этапам онтогенеза, в частности феномену привязанности, который определяет последующее развитие эго и метакогнитивных способностей [2]. Кожные заболевания можно рассматривать как общепсихологическую модель дифференциации и интеграции, как механизм, лежащий в основе построения концептуального знания [3].

Кожа имеет непосредственное отношение как к индивидному, так и к «социальному» уровням психического. «Кожа является органом, который регулирует первые интеракции и отношения; таким образом, кожа в значительной степени связана с до- и невербальным опытом и имплицитной памятью» [4, с. 237]. Некоторые кожные маркеры (татуировки, шрамирование, пирсинг) являются индикаторами групповой принадлежности.

Например, кожные самоповреждения доминируют как средство маркирования в подростковом возрасте, являются средством коммуникации, осуществляемым «деструктивно и болезненно, в то время как поглаживания кожи являются средством самоуспокоения» $[5$, c. 100$]$.

Неоспоримым фактом в настоящее время считается утверждение о том, что ранние отношения матери и младенца являются критическими для последующего психического развития. Эти взаимодействия включают поверхность тела, кожу и «создают ощущение ограниченности внутреннего пространства с помощью границы, которая переживается как кожа» [6, с. 177].

Э. Бик ввела понятие «психическая кожа». С помощью понятия «вторая кожа» описывается «защитная структура личности, которая обеспечивает псевдонезависимый modus vivendi - мускульный или интеллектуальный панцирь, при котором допускается самоконтейнирование, но отрицается нужда во внешнем объекте» [7, с. 209]. Психическая кожа описывается как «общая», или неразделенная между матерью и младенцем. «Способность матери реагировать на переживания своего ребенка, по-видимому, ощущается ребенком сначала как собирание воедино его телесных ощущений, порождающее начало ощущения телесной целостности» [8, с. 31]. Таким образом, кожа, согласно Э. Бик и ее последователей, выполняет функцию связывания («слипания») частей личности ребенка, которые еще не отдифференцированы как от частей тела младенца, так и матери. «Психическая кожа обеспечивает контейнирование психического материала младенца и выполняет защитную функцию» [9, с. 157]. Опыт контейнирования ведет к «формированию первичной кожи и создает „подмостки“ для последующего психологического развития» [6, с. 181]. Этот опыт может быть описан как «чувство телесной интеграции», «обладание кожей», «физическое ощущение себя» [8, с. 32].

В том случае если состояния или переживания младенца оказываются «неотраженными» (или, в терминах У. Биона, несконтейнированными), то возникает ощущение хаоса. Опыт, который переживает младенец в ранних симбиотических отношениях с матерью, является своего рода прототипом для последующих моделей взаимодействия человека с окружающим миром. Согласно У. Биону, «модель переработки эмоционального опыта если он многократно повторяется в опыте переживаний - становится фундаментальным фактором в процессе структурирования личности» [10, с. 24]. «Здоровое» ощущение себя во многом базируется на идентификации с интернализированным опытом хорошей матери, что «позволяет ребенку в будущем быть терпимым и открытым как внутреннему, так и внешнему опыту» [8, с. 36].

На ранних этапах развития - в ситуации первичного симбиоза, т. е. в состоянии слияния с матерью, я еще не выделилось из не-я. Процесс выделения и ментализации «внутри» 
и «снаружи» лишь постепенно приводит к ощущению себя как кого-то, отличного от другого. В тесной сопряженности с этим процессом происходит выделение собственного телесного я и выделение (ментализация) «другого» как живого существа.

В случае если мать способна «контейнировать» психический недифференцированный опыт младенца, то стадия первичного симбиоза проходит нормально. «По мере того как внутренний мир ребенка становится более интегрированным, становится возможным чувство внутренней континуальности. Около года ребенок может поддерживать внутренние отношения с матерью даже при ее отсутствии» [8, с. 45].

Если ребенок сталкивается с ранним травмирующим опытом материнского «непонимания», то он оказывается неспособным к интеграции «того, что внутри, и того, что происходит вовне». Как следствие, у младенца не возникает «чувства когерентности внутреннего и внешнего опыта» [11, с. 46]. Как указывает М. Вэдделл, «неурядицы» в отношениях матери и ребенка оставляют в характере человека трещины, такие же, как «период засухи оставляет отметины в куске древесины» [10, с. 20]. Поэтому истоки способности к сепарации, по словам автора, лежат в ранних отношениях «мать - ребенок». Нарушения в этих отношениях «ведут к эмоциональным нарушениям», в особенности к страху сепарации и «к тенденции защищаться от этих страхов с помощью разных психологических механизмов» $[10$, c. 160].

В том случае «если нормальная симбиотическая континуальность между матерью и младенцем прерывается из-за травматических обстоятельств», то, по словам А. Лемма, возникает «психическая абортация» [12]. Растерянность и утрата первичной континуальности «ведет к поиску разного рода других контейнеров», которые могут восполнить чувство интегрированности [12, с. 157]. Одним из способов такой деструктивной саморегуляции являются самоповреждения [5]. Данные психопатологии свидетельствуют о том, что есть два «защитных» выхода из этой ситуации неудавшейся сепарации - «примитивная инкапсуляция или интрузивная идентификация с другими» [13, с. 154]. И в том и в другом случае происходит деформация в процессе формирования подлинно автономного я.

Многие авторы рассматривают психосоматические заболевания как проявления кризисов, вызванных разного рода травматическими обстоятельствами [14, с. 111]), как своеобразную форму защиты истинного я (в случае если все другие способы защиты оказались тщетными). Развивается так называемая вторая кожа, т. е. ребенок развивает преждевременную независимость от фигуры первичной привязанности (матери) и «на поверхности проявляется как функционирующий в автономной манере» [6, с. 182]. Вторая кожа может формироваться при любых фрустрациях, например при переходе в подростковый возраст, «когда требуется переработать старые инфантильные конфликты» или когда встает задача «интернализации прежних объектных отношений и идентификаций» $[10$, с. 78]. В случае формирования второй, или ложной, кожи «ребенок развивает преждевременную независимость, и может показаться, что он функционирует автономно. Эта преждевременная автономия может маскировать чувство чрезвычайной тревоги, экстремальные формы избегающей привязанности и использовать автосенсорное поведение для самоуспокоения и комфорта» $[15$, с. 204]. Вторая кожа тормозит «психологическое и эмоциональное развитие через экстенсивное использование телесных защит» [6, с. 179].

Если ребенок не уверен в матери, то происходит нарушение «доверительной» связи, и в этом случае отщепленные части эго строят «новую защиту - вторичный симбиоз, который замораживает ментальную эволюцию этой отщепленной части» [12, с. 115]. Эта отщепленная структура эго «выживает как замороженная часть, и никакая психическая трансформация с ней не происходит по мере ее взросления» [12, с. 115]. 
А. Лемма связывает формирование второй кожи и вторичного симбиоза с тем, как мать смотрит на младенца. Автор выделяет два взгляда матери - невидящий и искажающий. На основании проведенных исследований на данной выборке испытуемых с помощью методики «Я-структурный тест Аммона» (ISTA) нами было доказано, что данные группы различаются по внутренним и внешним я-отграничениям. Мы доказали, что в том случае, когда младенец сталкивается с невидящим взором матери, он испытывает потребность «найти мать», т. е. обрести тот контейнер, который может структурировать его опыт, оставаясь тем самым в «вечной зависимости» от матери. Этот способ поведения характерен для группы «атопический дерматит». В случае если ребенок имел опыт искажающего взора матери, то он стремится «избежать» любого рода интимных контактов; этот способ поведения характерен для группы «псориаз» [16].

В данном исследовании мы полагаем, что две группы различаются по способу дифференциации «себя» и «другого».

Теоретической гипотезой исследования является предположение о том, что кожные заболевания являются формой разрешения сепарационного конфликта в виде вторичного симбиоза. В группе «атопический дерматит» выражены дефекты, сопряженные с затянувшимся симбиозом (т. е. с невидящим взором матери), а в группе «псориаз»- с несостоявшимся или преждевременно разорванным симбиозом (т. е. с искажающим взглядом матери).

Эмпирическая гипотеза: имеется сопряженная связь между способами репрезентации собственного я (с опорой на себя/на мнения другого) у женщин, страдающих хроническими кожными заболеваниями (псориазом и атопическим дерматитом), по сравнению с контрастной группой здоровых женщин.

Задачи исследования: выявление связей сопряженности между репрезентацией истинного я и предпочтениями в выборе крема в разных группах испытуемых.

Методы исследования:

- опросник «Диагностика телесного я» (шкалы: «Самопринятие своего телесного я», «Влияние другого на отношение к образу телесного я») [17];

- методика «Крем» [18].

Испытуемые: в исследовании участвовало 72 женщины в возрасте 20-40 лет, медиана 37 лет (30 - здоровых (НОРМ), 17 - страдающих псориазом (ПС) и 25 - атопическим дерматитом (АД)).

Исследование проводилось индивидуально на добровольной основе. При обработке результатов использовался метод Краскала - Уоллиса. Обработка данных проводилась с помощью пакета Statictica 8.0. Использовался непараметрический критерий Краскала Уоллиса.

Результаты множественного сравнения по критерию Краскала - Уоллиса приведены ниже.

Таблица 1

Зависимость переменной «Самопринятие своего телесного я» от выборки

\begin{tabular}{|c|c|c|c|}
\hline Группа & $\mathbf{1}-\mathbf{R : 4 3 , 2 3 3}$ & $\mathbf{2}-\mathbf{R : 1 6 , 6 2 0}$ & $\mathbf{3}-\mathbf{R : 5 3 , 8 5 3}$ \\
\hline НОРМ & - & $\mathbf{0 , 0 0 0}$ & 0,284 \\
\hline АД & $\mathbf{0 , 0 0 0}$ & - & 0,000 \\
\hline ПС & 0,284 & $\mathbf{0 , 0 0 0}$ & - \\
\hline
\end{tabular}

Примечание. В заголовке таблицы представлены значения рангов для каждой подгруппы испытуемых, в клетках таблицы приведены оценки вероятностей. Значимые отличия выделены жирным шрифтом.

Группа ПС достоверно оличается по переменной «Самопринятие своего телесного я» от группы АД и не различается с группой $\mathrm{HOPM}(\mathrm{H}(2, \mathrm{~N}=72)=38,46452 ; \mathrm{p}=0,0000)$. 
Таким образом, группа ПС (по сравнению с группой АД) больше опирается на конструкт «самопринятие своего телесного я». Достоверных различий по данному параметру между группой НОРМ и ПС нет.

Таблица 2

Зависимость переменной «влияние другого на отношение к образу телесного я» от выборки

\begin{tabular}{|c|c|c|c|}
\hline Группа & $\mathbf{1}-\mathbf{R : 1 6 , 0 3 3}$ & $\mathbf{2}-\mathbf{R : 5 9 , 3 8 0}$ & $\mathbf{3}-\mathbf{R : 3 8 , 9 7 1}$ \\
\hline НОРМ & - & $\mathbf{0 , 0 0 0}$ & $\mathbf{0 , 0 0 1}$ \\
\hline АД & $\mathbf{0 , 0 0 0}$ & - & $\mathbf{0 , 0 0 6}$ \\
\hline ПС & $\mathbf{0 , 0 0 1}$ & $\mathbf{0 , 0 0 6}$ & - \\
\hline
\end{tabular}

Примечание. В заголовке таблицы представлены значения рангов для каждой подгруппы испытуемых, в клетках таблицы приведены оценки вероятностей. Значимые отличия выделены жирным шрифтом.

Получено достоверное различие между всеми группами по переменной «влияние другого на отношение к образу телесного я» $(\mathrm{H}(2, \mathrm{~N}=72)=59,35715 ; \mathrm{p}=0,0000)$. Группа НОРМ показывает наименьшие результаты, группа АД - максимальные.

Таблица 3

Зависимость переменной «защчита» (методика «Крем») от выборки

\begin{tabular}{|c|c|c|c|}
\hline Группа & $\mathbf{1 - R : 2 1 , 3 1 7}$ & $\mathbf{2}-\mathbf{R : 5 6 , 0 2 0}$ & $\mathbf{3}-\mathbf{R : 3 4 , 5 8 8}$ \\
\hline НОРМ & - & $\mathbf{0 , 0 0 0}$ & 0,110 \\
\hline АД & 0,000 & - & 0,003 \\
\hline ПС & 0,110 & $\mathbf{0 , 0 0 3}$ & - \\
\hline
\end{tabular}

Примечание. В заголовке таблицы представлены значения рангов для каждой подгруппы испытуемых, в клетках таблицы приведены оценки вероятностей. Значимые отличия выделены жирным шрифтом

Получено достоверное различие между группой НОРМ и группой АД, а также группой АД и группой ПС по переменной «защита» $(\mathrm{H}(2, \mathrm{~N}=72)=40,84839 ; \mathrm{p}=0,0000)$. Группа НОРМ показывает наименьшие результаты, группа АД - максимальные.

Таблица 4

Зависимость переменной «очищение» (методика «Крем») от выборки

\begin{tabular}{|c|c|c|c|}
\hline Группа & $\mathbf{1 - R : 2 2 , 2 1 7}$ & $\mathbf{2 - R : 3 5 , 6 4 0}$ & $\mathbf{3}-\mathbf{R : 6 2 , 9 7 1}$ \\
\hline НОРМ & - & 0,053 & $\mathbf{0 , 0 0 0}$ \\
\hline АД & 0,053 & - & $\mathbf{0 , 0 0 0}$ \\
\hline ПС & $\mathbf{0 , 0 0 0}$ & $\mathbf{0 , 0 0 0}$ & - \\
\hline
\end{tabular}

Примечание. В заголовке таблицы представлены значения рангов для каждой подгруппы испытуемых, в клетках таблицы приведены оценки вероятностей. Значимые отличия выделены жирным шрифтом

Получено достоверное различие между группой НОРМ и группой ПС, а также между группами АД и ПС по переменной «защита» $(\mathrm{H}(2, \mathrm{~N}=72)=42,98498 ; \mathrm{p}=0,0000)$. Группа НОРМ показывает наименьшие результаты, группа ПС - максимальные.

По другим категориям методики «Крем» достоверных различий не получено.

Получен результат, свидетельствующий о том, что экспериментальные группы различаются в опоре на конструкты при описании собственного я («самопринятие своего телесного я»/«влияние другого на отношение к образу телесного я» как между собой, так и с группой контраста (НОРМ). При этом группа АД максимально опирается на «мнения другого», а группа ПС - на свой телесный опыт. По методике «Крем» получены различия в выборе предпочтительных категорий крема. Группа АД достоверно чаще выбирает категорию крема «защита», а группа ПС - «очищение». 
Согласно методике «Крем» [17], выбор категории «защита» положительно коррелирует с «эмоциональным способом совладающего поведения» и отрицательно - со стратегией «избегание» (методика выхода из конфликтных ситуаций Томаса).

Выбор категории «очищение», согласно методике, коррелирует с копинговой стратегией «социальное отвлечение» и с компромиссом как способом преодоления конфликтов.

Обобщенный портрет, основанный на полученных данных в отношении группы АД, можно описать следующим образом: выраженное доминирование мнений другого в репрезентации собственного я, потребность в защите, эмоциональная реакция на стрессовую ситуацию и избегание конфликтов. Содержательно полученный портрет соответствует тенденции к слиянию. Вэдделл определяет эту тенденцию как «первобытный страх быть разорванным на части» [10, с. 63], оказаться без материнского «контейнера». Это переживание можно соотнести с опытом младенца, который проживает симбиоз с «невидящим» взором матери и который всеми силами «стремится быть увиденным».

Обобщенный портрет, основанный на полученных данных в отношении группы ПС, можно описать следующим образом: выраженное «самопринятие своего телесного я» при одновременном «социальном отвлечении в выборе копинговой стратегии и компромисса как способа преодоления конфликта. Содержательно полученный портрет соответствует тенденции к инкапсуляции. А. Лемма рассматривает понятие «униженность» для описания опыта младенца в том случае, когда он «отбрасывает от себя как отвратительные» те проекции, которые он «интроецировал» в отношении себя в случае «искажающего взора матери» [9, с. 96]. Ранний опыт «материнского отвержения» формирует вторую кожу в форме избегания и инкапсуляции.

Выводы:

1. Кожные заболевания (АД и ПС) отражают разные способы преодоления вторичного симбиоза.

2. При репрезентации собственного я группа АД демонстрирует тенденцию к слиянию, а группа ПС - к избеганию.

\section{Список литературы}

1. Pally R. How Brain Development is shaped by Genetic and Environmental factors: Developments in related fields neurosciences // International Journal of Psycho-analysis. 1997. Vol. 78. P. 71-84.

2. Steele M. Attachment, actual experience and mental representation // Emotional development in psychoanalysis, attachment theory and neuroscience. Creating connections / ed. V. Green. Hove: Bruner-Routledge, 2003. P. 86-105.

3. Холодная М. А. Психология интеллекта. Парадоксы исследования: учеб. пособие для бакалавриата и магистратуры. М.: Юрайт, 2019. 334 c.

4. Ribeiro-Blanchard A. J., Seixas L. P., Rios F. M. G. The body in psychotherapy // Body, Mind and Healing after Jung / ed. Jones R. A. London; New York: Routledge, 2011. P. 228-249.

5. Campbell D., Hale R. Working in the dark. Understanding the pre-suicide state of mind. London; New York: Routledge, 2017. $119 \mathrm{p}$.

6. Feldman B. Creating a skin for imagination, reflection, and desire /Transformation. Jung's legality and clinical work today / ed. A. Cavalli, L. Hawkins, M. Stevns. London; New York: Routledge, 2014. P. 177-192.

7. Jackson J., Nowers E. The skin in early object relations revisited/Surviving space. Papers on infant observation / ed. A. Briggs. London: Karnac, 2002. P. 208-239.

8. Shuttleworth J. Psychoanalytic Theory and Infant Development // Closely observed Infants / ed. L. Miller, M. Rustin, J. Shuttleworth. Duckworth, 2002. P. 22-51.

9. McAlister M. Beneath the skin: archetypal activity in Psychosis // Transformation. Jung's legality and clinical work today / ed. A. Cavalli, L. Hawkins, M. Stevns. London; New York: Routledge, 2014. P. 155-173.

10. Waddell M. On adolescence. Inside Stories. London: Karnac, 2018. 282 p. 
11. Waddell M. Infancy: defenses against pain // Psychotic states in children // ed. M. Rustin, M. Rhode, H. Dubinsky, A. Dubinsky. London; New York: Karnac, 1997. P. 45-59.

12. Lemma A. Under the skin. A psychoanalytic study of body modification. London; New York: Routledge, 2010. 205 p.

13. Bergstein A. Bion and Meltzer's expeditions into unmapped mental life. Beyond the Spectrum in Psychoanalysis. London; New York: Routledge, 2019. 203 p.

14. Lutenberg J. M. Mental void and the borderline patient // Resonance of suffering. Countertransference in Non-Neurotic structures / ed. A. Green. London: Karnac, 2007. P. 89-120.

15. Cavalli A. From not knowing to knowing: on early infantile trauma involving separation // Transformation. Jung's legacy and clinical work today / ed. A. Cavalli, L. Hawkins, M. Stevns. London; New York: Routledge, 2014. P. 193-210.

16. Лыбко И. В. Методика «Диагностика телесного я» // Психологическая диагностика: научно-методический и практический журнал. 2008. № 3. Р. 5-21.

17. Ребеко Т. А. Отношение к коже как имплицитная модель фемининности // Вестн. Российского гуманитарного научного фонда. 2006. № 1 (42). Р. 167-180.

Ребеко Татьяна Анатольевна, кандидат психологических наук, ведущий научный сотрудник, Институт психологии РАН (ул. Ярославская, 13, Москва, 129366). E-mail: rebekota@yandex.ru

Материал поступил в редакиию 08.11.2019.

DOI 10.23951/2307-6127-2020-1-174-181

\section{PSYCHOSOMATIC DISEASES AS THE SEARCH FOR SELF}

\section{T. A. Rebeko}

Institute of Psychology of Russian Academy of Sciences, Moscow, Russian Federation

The problem of psychosomatic diseases is discussed. Skin diseases are considered as a General psychological model of differentiation and integration of knowledge about the self. The paper investigates the conjugate relationship between psychosomatic skin diseases (psoriasis and atopic dermatitis) and the representation of the self. The literature data in favor of the statement that the early relationship of mother and baby are critical for subsequent mental development. The concept of "primary skin" (E. Bick), which performs the function of binding ("sticking together") parts of the child's personality, is considered. It is suggested that the experience passed through by the infant in the early symbiotic relationship with the mother is a prototype for subsequent models of human interaction with the world. If a child is confronted with an early traumatic experience of maternal "misunderstanding," then he is unable to integrate "what is inside and what is happening outside." The concepts of "second skin" and secondary symbiosis as protective mechanisms arising in the case of traumatic separation experience are considered. The forms of secondary symbiosis are correlated with two types of the mother's gaze- the unseeing and the distorting. It is assumed that the two types of gazes and forms of secondary symbiosis correlate with atopic dermatitis and psoriasis. It is hypothesized that defects in the "atopic dermatitis" group are associated with prolonged symbiosis (that is, with the mother's unseeing gaze), and in "psoriasis" group with defective or prematurely torn symbiosis (i. e. distorting mother's gaze). The study was carried out using the Lybko technique (self-representation by means of constructs Myself/I for the Other) and the "Cream" technique (representation of the skin surface by means of different constructs of the cream). The study involved 72 women aged 20-40 years, IU $=37$ (30 - healthy (NORMAL), 17 suffering from psoriasis (PS) and 25 - atopic dermatitis (AD)). The method of nonparametric analysis (Kruskal - Wallis criterion) proves that the representation of the true Self manifests itself as a tendency to merge/encapsulate in different groups of subjects. Skin diseases (AD and PS) reflect different ways to overcome secondary 
symbiosis. When representing the Self, the AD group shows a tendency to fusion, and the PS group shows a tendency to encapsulation.

Keywords: self, psychic skin, second skin, primary/secondary symbiosis, atopic dermatitis/psoriasis, tendency to fusion/encapsulation.

\section{References}

1. Pally R. How Brain Development is shaped by Genetic and Environmental factors: Developments in related fields neurosciences. International Journal of Psycho-analysis, 1997, vol. 78, pp. 71-84.

2. Steele M. Attachment, actual experience and mental representation. Emotional development in psychoanalysis, attachment theory and neuroscience. Creating connections. Ed. V. Green. Hove, Bruner-Routledge, 2003. Pp. 86-105.

3. Kholodnaya M. A. Psikhologiya intellekta. Paradoksy issledovaniya: uchebnoye posobiye dlya bakalavriata i magistratury [Psychology of intelligence. Paradoxes of research: textbook for undergraduate and graduate studies]. Moscow, Yurayt Publ., 2019. 334 p. (in Russian).

4. Ribeiro-Blanchard A. J., Seixas L. P., Rios F. M. G. The body in psychotherapy. Body, Mind and Healing after Jung. Ed. R. A. Jones. London; N.Y., Routledge, 2011. Pp. 228-249.

5. Campbell D., Hale R. Working in the dark. Understanding the pre-suicide state of mind. London; N.Y., Routledge, 2017. 119 p.

6. Feldman B. Creating a skin for imagination, reflection, and desire. Transformation. Jung's legality and clinical work today. Ed. A. Cavalli, L. Hawkins, M. Stevns. London: N.Y., Routledge, 2014. Pp. 177-192.

7. Jackson J., Nowers E. The skin in early object relations revisited. Surviving space. Papers on infant observation. Ed. A. Briggs. London, Karnac, 2002. Pp. 208-239.

8. Shuttleworth J. Psychoanalytic Theory and Infant Development. Closely observed Infants. Ed. L. Miller, M. Rustin, J. Shuttleworth. Duckworth, 2002. Pp. 22-51.

9. McAlister M. Beneath the skin: archetypal activity in Psychosis. Transformation. Jung's legality and clinical work today. Ed. A. Cavalli, L. Hawkins, M. Stevns. London; N.Y., Routledge, 2014. Pp. 155-173.

10. Waddell M. On adolescence. Inside Stories. London, Karnac, 2018. 282 p.

11. Waddell M. Infancy: defenses against pain. Psychotic states in children. Ed. M. Rustin, M. Rhode, H. Dubinsky, A. Dubinsky. London; N.Y., Karnac, 1997. Pp. 45-59.

12. Lemma A. Under the skin. A psychoanalytic study of body modification. London; N.Y., Routledge, 2010. 205 p.

13. Bergstein A. Bion and Meltzer's expeditions into unmapped mental life. Beyond the Spectrum in Psychoanalysis. London; N.Y., Routledge, 2019. 203 p.

14. Lutenberg J. M. Mental void and the borderline patient. Resonance of suffering. Countertransference in Non-Neurotic structures. Ed. A. Green. London, Karnac, 2007. Pp. 89-120.

15. Cavalli A. From not knowing to knowing: on early infantile trauma involving separation. Transformation. Jung's legacy and clinical work today. Ed. A. Cavalli, L. Hawkins, M. Stevns. London; N.Y., Routledge, 2014. Pp. 193-210.

16. Lybko I. V. Metodika "Diagnostika telesnogo ya" [Method "Diagnostics of bodily I"]. Psikhologicheskaya diagnostika: nauchnometodicheskiy i prakticheskiy zhurnal, 2008, no. 3, pp. 5-21 (in Russian).

17. Rebeko T. A. Otnosheniye k kozhe kak implitsitnaya model' femininnosti [Attitude to the skin as an implicit model of femininity]. Vestnik Rossiyskogo gumanitarnogo nauchnogo fonda - Bulletin of The Russian Humanitarian Science Foundation, 2006, no. 1 (42), pp. 167-180 (in Russian).

Rebeko T. A., Institute of Psychology of Russian Academy of Sciences (ul. Yaroslavskaya, 13, Moscow, Russian Federation, 129366). E-mail: rebekota@yandex.ru 